\title{
I think, therefore I am (unhappy)
}

\section{Juergen Fell*}

Department of Epileptology, University of Bonn, Bonn, Germany

*Correspondence: juergen.fell@ukb.uni-bonn.de

When not performing a task, we are usually engaged in a variety of thoughts, for instance about current problems, opportunities, our self-image, our relationships, about past experiences, and about plans for the future. Such mind wandering was found to occupy almost half of our time spent awake (Killingsworth and Gilbert, 2010). Mind wandering may have had the evolutionary advantage of better adaptation to changing environmental and social circumstances, because it allows the mental contemplation of issues remote from the here and now (Schooler et al., 2011).

But mind wandering also has substantial downsides. Even when we are performing a task, our minds tend to wander. This divagation into situation-independent thoughts usually goes unnoticed and it leads to impaired task performance (e.g., Christoff et al., 2009; Schooler et al., 2011). Depending on the task, this actually may be dangerous, for instance when driving a vehicle (He et al., 2011). Furthermore, mind wandering is related to negative emotions (e.g., Smallwood et al., 2009). A recent study has demonstrated that people are less happy when their minds are wandering to neutral topics than when their minds are focused on their current activity (Killingsworth and Gilbert, 2010). And even when thinking about pleasant topics, they are not happier than when they focus on the task at hand (Killingsworth and Gilbert, 2010).

Knowledge about the negative effects of mind wandering has been available for a long time in various religious traditions, in particular in Buddhism. The practice of mindfulness meditation aims at reducing the prevalence of uncontrolled thought chains by increasing meta-awareness of ongoing mental activities. The finding that mind wandering related brain activations (see below) are diminished in case of meta-awareness (Christoff et al., 2009) provides neuroscientific validation for this strategy. Today, elements of mindfulness meditation have been implemented in several therapeutic systems, such as mindfulnessbased stress reduction (Ludwig and KabatZinn, 2008) and mindfulness-based cognitive therapy (Segal et al., 2002). These techniques are applied, for instance, for the treatment of attention deficit disorder, psychosomatic disorders, and depression.

But is there neuroscientific evidence that mindfulness training indeed reduces neural activations associated with mind wandering? Recent fMRI studies support this assumption. First of all, mind wandering was found to be associated with activations in so-called default mode brain areas (Mason et al., 2007; Christoff et al., 2009; Stawarczyk et al., 2011; Hasenkamp et al., 2012). These brain areas were demonstrated to exhibit increased activity during task-free periods and decreased activity during goaloriented behavior as measured by functional brain imaging (Raichle et al., 2001). The default mode network comprises, for instance, parts of medial prefrontal, medial temporal and parietal cortex, the posterior cingulate cortex, and the precuneus.

A recent study investigated activations in default mode areas in subjects with no, moderate, and high meditation experience during daydreaming and mindfulness meditation (Ott et al., 2010). The authors observed an inhibition of default mode network activity during mindfulness meditation compared to the daydreaming condition. Moreover, the inhibition of anterior default mode areas, which have been argued to be associated with self-related and social cognition (Northoff et al., 2006), was more persistent in subjects with high compared to no meditation experience.

In a similar vein, a recent investigation addressed the changes in brain activation during three types of mindfulness meditation in experienced meditators and meditation-naïve controls (Brewer et al., 2011). Compared to the controls experienced meditators reported less mind wandering during the meditation periods and the main nodes of the default mode network, medial prefrontal and posterior cingulate cortex, were deactivated across all meditation types. Moreover, functional connectivity analyses revealed in the experienced meditators versus controls increased coactivation of the posterior cingulate and the dorsal anterior cingulate (during resting state baseline and meditation), as well as the dorsolateral prefrontal cortex (during baseline), i.e., regions probably involved in self-monitoring and cognitive control (Brewer et al., 2011). This result suggests that processes supported by the default mode network are more accessible to monitoring and control in experienced meditators. The authors discuss that control regions may coactivate to monitor and dampen processes related to the default mode network when interfering with a task. They speculate that this coactivation may, over time, become a new "default mode" that is established not only during meditation, but also during the resting state.

Another study addressed the neural correlates of conceptual processing in regular Zen (a particular kind of mindfulness meditation) practitioners compared to matched control subjects. Participants were required to decide whether sparsely presented visual stimuli were real English words or strings of letters with plausible readings but no semantic content (Pagnoni et al., 2008). The words, compared to the non-words, are likely to trigger a cascade of semantic associations. Moreover, participants were instructed to attend to their breathing throughout the scan and to promptly re-focus attention to their breathing after responding to the stimuli. The authors found that Zen practitioners, compared to controls, displayed a reduced duration of semantic processing effects in default mode brain areas suggesting greater ability to disengage from mind wandering.

Furthermore, one investigation evaluated functional activations during two tasks associated with reading trait related adjectives (Farb et al., 2007). In one task, subjects should reflect on what the adjective meant about them as a person (narrative focus), while in the other task they should monitor their moment-to-moment experience in response to the adjectives (experiential focus). The authors recruited participants of an 8-week course in mindfulness meditation and novices. During the experiential compared to the narrative condition reductions 
in self-referential cortical midline regions were observed. In the medial prefrontal cortex these reductions were more marked and pervasive in trained participants than in controls.

Finally, a recent study investigated the neural substrates of mindfulness in subjects who had undergone a mindfulness-based stress reduction intervention and had practised mindfulness daily for a minimum of 4 years (Ives-Deliperi et al., 2011). Activations during mindfulness meditation were compared to a number-generation control task. Again, the authors observed mindfulness-related signal decreases mainly in cortical midline structures, which are known to be related to interoception and self-related mental processes and are part of the default mode network.

To conclude, several recent fMRI studies revealed reduced neural activations within default mode brain areas during mindfulness practice and these effects appear to be most pronounced in experienced meditators. These data represent preliminary neuroscientific support for the idea that mindfulness training is an efficient method to reduce neural processes associated with mind wandering and task-independent thoughts. However, additional studies are needed to corroborate these initial findings. Furthermore, it is still unclear whether mindfulness-related alterations of neural activations are directly linked to modifications of behavior and whether mindfulness training can indeed induce persistent changes in the default mode network.

\section{ACKNOWLEDGMENTS}

The author wants to thank Nikolai Axmacher, Ulrich Ott, Bernhard Staresina, and an anonymous referee for helpful comments and suggestions.

\section{REFERENCES}

Brewer, J. A., Worhunsky, P. D., Gray, J. R., Tang, Y. Y., Weber, J., and Kober, H. (2011). Meditation experience is associated with differences in default mode network activity and connectivity. Proc. Natl. Acad. Sci. U.S.A. 108, 20254-20259.

Christoff, K., Gordon, A. M., Smallwood, J., Smith, R., and Schooler, J. W. (2009). Experience sampling during fMRI reveals default network and executive system contribution to mind wandering. Proc. Natl. Acad. Sci. U.S.A. 106, 8719-8724.

Farb, N. A., Segal, Z. V., Mayberg, H., Bean, J., McKeon, D., Fatima, Z., and Anderson, A. K. (2007). Attending to the present: mindfulness meditation reveals distinct neural modes of self-reference. Soc. Cogn. Affect. Neurosci. 2, 313-322.

Hasenkamp, W., Wilson-Mendenhall, C. D., Duncan, E., and Barsalou, L. W. (2012). Mind wandering and attention during focused meditation: a fine-grained temporal analysis of fluctuating cognitive states. Neuroimage 59, 750-760.

He, J., Becic, E., Lee, Y. C., and McCarley, J. S. (2011). Mind wandering behind the wheel: performance and oculomotor correlates. Hum. Factors 53, 13-21.

Ives-Deliperi, V.L., Solms, M., and Meintjes, E. M. (2011) The neural substrates of mindfulness: an fMRI investigation. Soc. Neurosci. 6, 231-242.

Killingsworth, M. A., and Gilbert, D. T. (2010). A wandering mind is an unhappy mind. Science 330, 932.

Ludwig, D. S., and Kabat-Zinn, J. (2008). Mindfulness in medicine. JAMA 300, 1350-1352.

Mason, M. F., Norton, M. I., Van Horn, J. D., Wegner, D. M., Grafton, S. T., and Macrae, C. N. (2007). Wandering minds: the default network and stimulusindependent thought. Science 315, 393-395.
Northoff, G., Heinzel, A., de Greck, M., Bermpohl, F. Dobrowolny, H., and Panksepp, J. (2006). Self-related processing in our brain - a meta-analysis of imaging studies on the self. Neuroimage 31, 440-457.

Ott, U., Walter, B., Gebhardt, H., Stark, R., and Vaitl, D. (2010). "Inhibition of default mode network activity during mindfulness meditation," in HBM 2010, Barcelona.

Pagnoni, G., Cekic, M., and Guo, Y. (2008). “Thinking about not-thinking": neural correlates of conceptual processing during Zen meditation. PLoS ONE 3, e3083. doi: 10.1371/journal.pone.0003083

Raichle, M. E., MacLeod, A. M., Snyder, A. Z., Powers, W. J., Gusnard, D. A., and Shulman, G. L. (2001). A default mode of brain function. Proc. Natl. Acad. Sci. U.S.A. 98, 676-682.

Schooler, J. W., Smallwood, J., Christoff, K., Handy, T. C., Reichle, E. D., and Sayette, M. A. (2011). Metaawareness, perceptual decoupling and the wandering mind. Trends Cogn. Sci. (Regul. Ed.) 15, 319-326.

Segal, Z., Teasdale, J., and Williams, M. (2002). Mindfulness-Based Cognitive Therapy for Depression. New York: Guilford Press.

Smallwood, J., Fitzgerald, A., Miles, L. K., and Phillips, L. H. (2009). Shifting moods, wandering minds: negative moods lead the mind to wander. Emotion 9, 271-276.

Stawarczyk, D., Majerus, S., Maquet, P., and D'Argembeau, A. (2011). Neural correlates of ongoing conscious experience: both task-unrelatedness and stimulus-independence are related to default network activity. PLoS ONE 6, e16997. doi: 10.1371/journal.pone.0016997

Received: 27 October 2011; accepted: 25 April 2012; published online: 16 May 2012.

Citation: Fell J (2012) I think, therefore I am (unhappy). Front. Hum. Neurosci. 6:132. doi: 10.3389/ fnhum.2012.00132

Copyright $\odot 2012$ Fell. This is an open-access article distributed under the terms of the Creative Commons Attribution Non Commercial License, which permits non-commercial use, distribution, and reproduction in other forums, provided the original authors and source are credited. 